\title{
Ghanges in Gross Chemical Components of Trichophyton mentagrophytes During Incubation in Increased Carbon Dioxide Tensions
}

\author{
By B. CHIN and S. G. KNIGHT \\ Department of Bacteriology, University of Wisconsin, Madison, Wisconsin, U.S.A
}

(Received 21 May 1962)

\begin{abstract}
SUMMARY
Macroconidia of Trichophyton mentagrophytes harvested from a culture grown on a medium rich in glucose contained $33 \%$ acid-soluble carbohydrate. Paper chromatography of hydrolysates showed glucose as the main sugar in this fraction; there was also a trace of an unidentified sugar. Macroconidia from the same strain grown on the same medium but deficient in glucose contained $19 \%$ carbohydrate; hydrolysates of this fraction contained glucose, with traces of galactose and the unidentified sugar. Microconidia harvested from a second strain grown on a medium rich in glucose contained $11 \%$ acid-soluble carbohydrate; hydrolysates of this fraction also contained glucose with traces of galactose and the unidentified sugar. These differences were reflected in whole cultures induced for macroconidial formation with carbon dioxide. Strains which responded well increased in acid-soluble carbohydrate; glucose was the predominating sugar in hydrolysates of these fractions. Strains which responded poorly did not increase in carbohydrate content; glucose, and traces of galactose and an unidentified sugar, were found in hydrolysates of these fractions.
\end{abstract}

\section{INTRODUCTION}

A previous report from this laboratory (Chin \& Knight, 1957) described the stimulation of macroconidial formation in Trichophyton mentagrophytes during incubation at increased carbon dioxide tensions. A requirement for glucose was demonstrated, since macroconidia formation was not stimulated in increased atmospheric tensions of $\mathrm{CO}_{2}$ when glucose was omitted from the medium. The experiments described in the present paper attempted to analyse and compare the gross chemical components of macroconidia, microconidia and hyphae. It was our intention to find some measurable difference between macroconidia and the other morphological units of $T$. mentagrophytes with the hope that this difference might be induced in the normally non-sporulating strains when macroconidia formation was stimulated by carbon dioxide. Because of difficulties involved in separating macroconidia from microconidia and hyphae, mutant strains which differed in type of sporulation were derived from a common parent. Macroconidia are easily harvested from a sporulating mutant which does not form microconidia, and vice versa. 
Mutants

METHODS

All mutants were derived from Trichophyton mentagrophytes strain A-280 (from the Communicable Disease Center, Chamblee, Georgia, U.S.A.). Stock cultures were maintained by 3-monthly transfer on Sabouraud's glucose agar (Difco) and incubation at $30^{\circ}$ for 10-14 days followed by storage at $5^{\circ}$. Sabouraud's glucose agar (4\% glucose, $1 \%$ Neopeptone, $1.5 \%$ agar) was used throughout. Liquid medium was prepared by omitting the agar, and Sabouraud's conservation medium by the omission of glucose. Cultures were incubated for 5 days before harvest. The genetic purities of parent and mutant strains were assured by single spore isolations to avoid the selection of macroconidia-forming variants from mixed clones during incubation in $\mathrm{CO}_{2}$ (Emmons \& Hollaender, 1939). A microconidium was isolated from strain A-280 by the excision and transfer to fresh medium of a germinated microconidium from ruled agar blocks of Sabouraud's glucose agar. The clone resulting from the growth of this spore was maintained as the parent culture, strain 5-7. Spores from this clone were seeded on Petri plates containing solid media and irradiated with ultraviolet radiation. Morphological mutants differing in type of sporulation were selected from the survivors and transferred to fresh medium. In turn, clones resulting from the growth of transferred survivors were purified by the isolation of microconidia. Since strain 1-2-1 does not form microconidia, a macroconidium was isolated. The growth characteristics of all mutants have remained stable through 3 years of serial transfer. Their sporulating patterns and responses to $\mathrm{CO}_{2}$ are recorded in Table 1 .

Table 1. Macroconidial formation by mutants of Trichophyton mentagrophytes and their response to increased carbon dioxide tensions in air

$\begin{array}{clc}\text { Mutant } & \text { Normal air } & 16 \%(\mathrm{v} / \mathrm{v}) \mathrm{CO}_{2} \\ 5-7 & \text { rare } & \text { profuse } \\ 1-1-2 & \text { none } & \text { poor } \\ 1-2-1 & \text { abundant } & \text { profuse } \\ 1-8-1 & \text { none } & \text { poor }\end{array}$

Inoculum and growth

Inoculum for solid media was prepared by dispersing the growth on a stock culture slope with sterile water and pipette. Cultures for chemical analysis were grown as surface mats on liquid media. A floating inoculum was prepared by pipetting $1 \mathrm{ml}$. medium on to the surface of a stock culture slope. The culture tube was rolled gently to dislodge spores and hyphal fragments, the majority of which floated on the surface of the medium. In inoculation, this preparation was poured into a flask containing liquid medium. Flasks which were incubated with increased atmospheric tensions of $\mathrm{CO}_{2}(0-16 \% \mathrm{v} / \mathrm{v})$ were fitted with rubber stoppers and glass tubing outlets. The gassing procedure was as previously described (Chin \& Knight, 1957). After incubation the floating cultures were washed by pouring off the media and refloating the culture mats with water 4 times. The mats were then removed from the flasks and suspended in cold acetone (Umbreit, Burris \& Stauffer, 1957). The organism was separated from the acetone by centrifugation and the supernatant acetone drawn off through a capillary pipette connected to an aspirator pump. Samples of organism were dried in vacuo overnight. 
Spores

Spores were harvested from cultures grown on solid media in Roux bottles; macroconidia were harvested from strain 1-2-1, microconidia from strain 5-7. After 5 days of incubation, $40 \mathrm{ml}$. water were added to each bottle, the contents vigorously shaken and strained through gauze. Although many spores were retained with mycelium in the filter, the effluent usually contained a high percentage of the spores in the original harvest. Spores were separated from the effluent by centrifugation. The spores were washed 4 times, after which they were suspended in cold acetone and dried as described above.

\section{Spore walls}

Macroconidia were harvested and broken, prior to acetone treatment, in a Serval Omni-mix with water and glass microbeads for $30 \mathrm{~min}$. The proportions of the mixture were spores + glass beads + water $(1+2+3$, by vol. $)$. Microscopic examination of samples stained with lactophenol cotton blue revealed that $95 \%$ of the macroconidia were broken in all compartments. The spore walls were washed with water in excess of twenty times, suspended in cold acetone, and dried.

\section{Extraction procedure}

Weighed samples were transferred to test tubes and sequentially extracted in a boiling water bath with water for $15 \mathrm{~min}$., with $5 \%(\mathrm{w} / \mathrm{v})$ trichloroacetic acid (TCA) for $30 \mathrm{~min}$., with $\mathrm{N}-\mathrm{NaOH}$ for $30 \mathrm{~min}$. The tubes were capped with glass marbles to prevent evaporation. After each extraction, the contents of the tubes were separated by centrifugation. Samples of extracts were removed for analysis with a pipetting bulb; the rest of the extract was discarded through a capillary pipette connected to an aspirator pump. The cell residues remaining after extraction with hot $\mathrm{NaOH}$ were washed with water four times by repeated centrifugation and transferred to tared aluminum cups. The residues were dried overnight at $80^{\circ}$ and the weights determined by difference.

\section{Chemical analyses}

Carbohydrate was measured with the anthrone reagent, with glucose as the standard (Loewus, 1952). Samples from TCA extracts were hydrolysed with $0.6 \mathrm{~N}-$ $\mathrm{HCl}$ in a boiling water bath before paper chromatography. The solvent systems used for paper chromatography were isopropanol + water $(8+2$, by vol.) and amyl acetate + pyridine + water $(\mathbf{3}+\mathbf{3}+\mathbf{1}$, by vol. $)$. Paper chromatograms were developed with $\mathrm{AgNO}_{3}$ reagent (Smith, 1958).

Protein was measured with the Folin phenol reagent method based on the modification of Lowry, Rosenbrough, Farr \& Randall (1951).

Total nucleic acids (NA) were estimated by the method of Logan, Mannell \& Rossiter (1952) at $268.5 \mathrm{~m} \mu$ in a Beckman model DU spectrophotometer with 3.0 ml. silica cuvettes after extraction by the method of Schneider (1945). Samples were read against a blank of $5 \%$ TCA. Herring sperm deoxyribonucleic acid (DN A Nutritional Biochemical Co.), similarly hydrolysed, was used as the standard.

DNA content was determined by the method of Kech (1958). Ribonucleic acid (RNA) was estimated by the difference between total NA and DNA. 


\section{RESULTS}

The gross chemical compositions of macroconidia, microconidia, and whole culture of Trichophyton mentagrophytes strain 5-7, which produces microconidia but no macroconidia on Sabouraud's glucose agar, are described in Table 2. The

Table 2. Gross chemical components of macroconidia, microconidia, and whole culture of strain 5-7 of Trichophyton mentagrophytes

\begin{tabular}{|c|c|c|c|c|}
\hline & & $\begin{array}{l}\text { Macro- } \\
\text { conidia }\end{array}$ & $\begin{array}{l}\text { Micro- } \\
\text { conidia } \\
\text { of dry }\end{array}$ & Strain 5-7 \\
\hline $\mathrm{H}_{2} \mathrm{O}$ & $\begin{array}{l}\text { CHO* } \\
\text { PRO }\end{array}$ & $\begin{array}{l}11 \cdot 3 \\
11 \cdot 5\end{array}$ & $\begin{array}{r}13 \cdot 2 \\
7 \cdot 3\end{array}$ & $\begin{array}{r}7 \cdot 6 \\
12 \cdot 3\end{array}$ \\
\hline TCA & $\begin{array}{l}\text { CHO } \\
\text { PRO } \\
\text { RNA } \\
\text { DNA }\end{array}$ & $\begin{array}{r}\mathbf{3 3 \cdot 3} \\
5 \cdot 5 \\
\mathbf{2 \cdot 6} \\
\mathbf{0 \cdot 2}\end{array}$ & $\begin{array}{r}11 \cdot 1 \\
5 \cdot 4 \\
3 \cdot 5 \\
0 \cdot 3\end{array}$ & $\begin{array}{r}11 \cdot 9 \\
5 \cdot 8 \\
1 \cdot 4 \\
0 \cdot 1\end{array}$ \\
\hline $\mathrm{NaOH}$ & $\begin{array}{l}\text { PRO } \\
\text { CR }\end{array}$ & $\begin{array}{l}15 \cdot 6 \\
10 \cdot 6\end{array}$ & $\begin{array}{l}16 \cdot 0 \\
12 \cdot 0\end{array}$ & $\begin{array}{l}13 \cdot 4 \\
30 \cdot 1\end{array}$ \\
\hline
\end{tabular}

* CHO: carbohydrate, PRO, protein; RNA, ribonucleic acid; DNA, deoxyribonucleic acid; CR, cell residue.

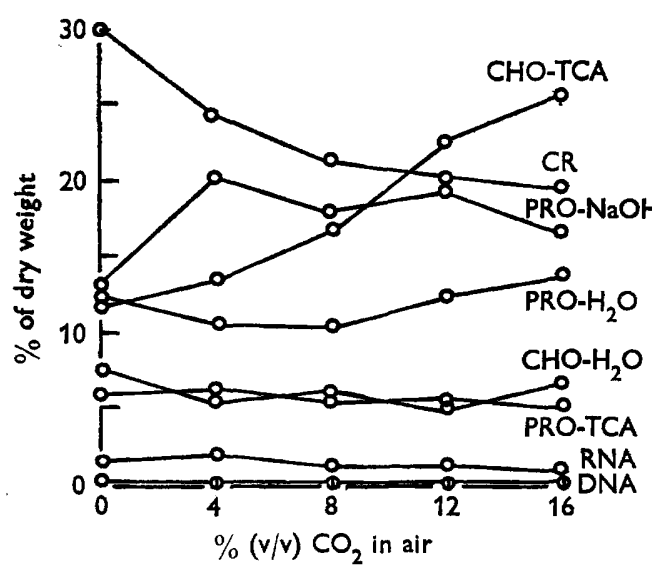

Fig. 1

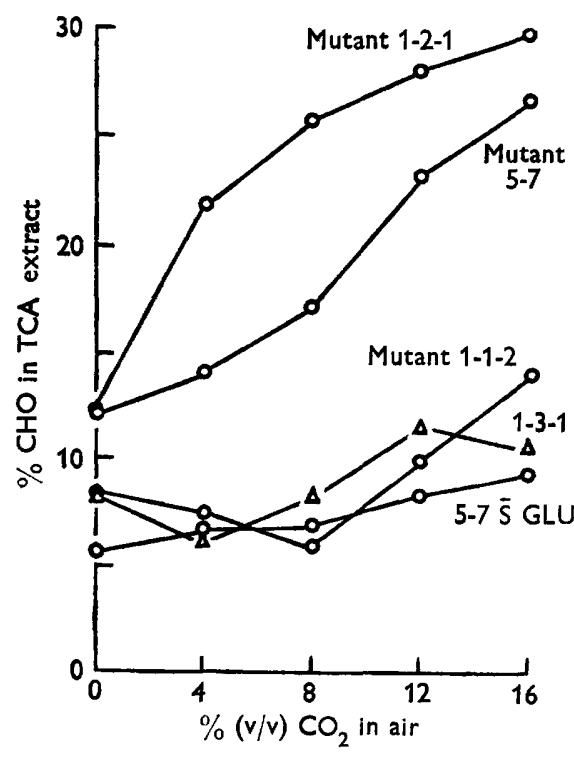

Fig. 2

Fig. 1. Changes in gross chemical composition, in percent of dry weight, of Trichophyton mentagrophytes strain 5-7 after incubation in increased carbon dioxide tensions. CHO$\mathrm{H}_{2} \mathrm{O}$, hot-water-extractable carbohydrate; $\mathrm{PRO}-\mathrm{H}_{2} \mathrm{O}$, hot-water-extractable protein; CHO-TCA, hot-TCA-extractable carbohydrate; PRO-TCA, hot-TCA-extractable protein; RNA, ribonucleic acid; DNA, deoxyribonucleic acid; $\mathrm{PRO}-\mathrm{NaOH}$, hot-NaOH-extractable protein; CR. cell residue.

Fig. 2. Changes in acid-soluble carbohydrate, in percent of dry weight, in mutants which vary in type and degree of sporulation during incubation in increasing carbon dioxide tensions. All mutants were harvested as surface cultures on liquid Sabouraud's medium, strain 5-7 was grown on medium with and without glucose ( $\overline{\mathrm{S}}$ GLU). 
macroconidia contained $\mathbf{3 3} \%$ hot-water-insoluble, hot-acid-soluble carbohydrate as compared with $11 \%$ in microconidia and $12 \%$ in strain $5-7$. These differences became more striking when paper chromatograms of hydrolysates of these fractions were examined. Glucose and trace amounts of an, at present, unidentified sugar were found in the hydrolysate of the TCA extract from macroconidia; in microconidia and whole organisms of strain 5-7, glucose and trace amounts of galactose and the same unidentified sugar were found. The unidentified sugar is suspected to be fructose, rhamnose or sorbose. Macroconidia harvested from strain 1-2-1 grown on Sabouraud's conservation agar (prepared by the omission of glucose) contained $19 \%$ acid-soluble carbohydrate. Again, paper chromatography showed that

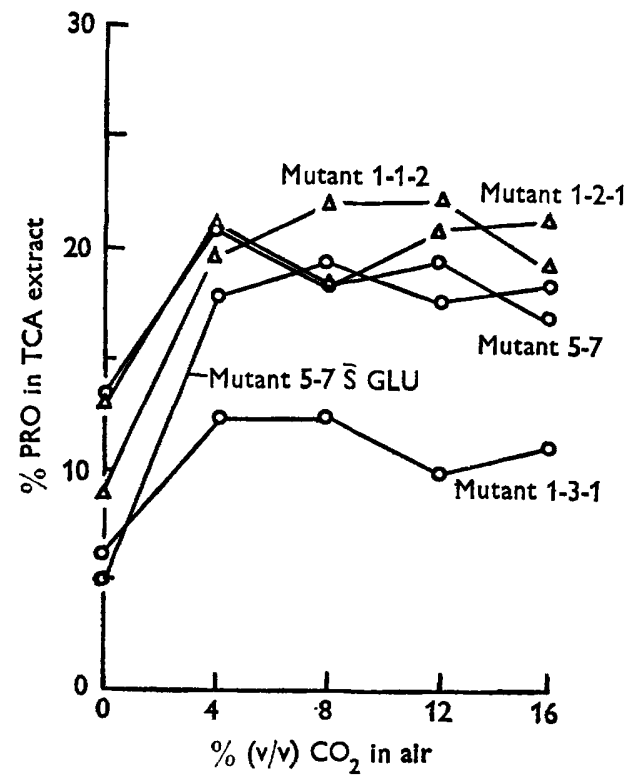

Fig. 3



Fig. 4

Fig. 3. Changes in protein content, in percent of dry weight, in mutants which vary in type and degree of sporulation during incubation in increasing carbon dioxide tensions. All mutants were harvested as surface cultures on liquid Sabouraud's medium; strain 5-7 was grown on medium with and without glucose ( $\overline{\mathbf{S}}$ GLU).

Fig. 4. Changes in cell residue, in percent of dry weight, in mutants which vary in type and degree of sporulation during incubation in increasing carbon dioxide tensions. All mutants were harvested as surface cultures on liquid Sabouraud's medium; strain 5-7 was grown on medium with and without glucose ( $\overline{\mathrm{S}}$ GLU).

glucose was the predominant sugar in hydrolysates of this fraction; only traces of galactose and the unidentified sugar were found. Walls of macroconidia from strain 1-2-1 grown on Sabouraud's glucose agar contained $9 \%$ acid-soluble carbohydrate.

After pre-treatment with acetone, the cytoplasm of macroconidia, microconidia and hyphae stain red-brown with Lugol's iodine solution. Hyphal and spore walls do not take the stain.

Strains 5-7 and 1-2-1 responded markedly to incubation in increased atmospheric tensions of $\mathrm{CO}_{2}$ and macroconidia formation was stimulated greatly. Strains 1-1-2 
and 1-3-1 did not respond well to $\mathrm{CO}_{2}$ and macroconidia formation was poorly stimulated. The changes in gross chemical components of the parent strain, 5-7, grown in increasing amounts of $\mathrm{CO}_{2}$ are shown in Fig. 1. The greatest fluctuations occur in three fractions: acid soluble carbohydrate (CHO-TCA), $\mathrm{NaOH}$-extractable protein (PRO-NaOH), and cell residue (CR). The changes in these fractions, for all mutants, in response to $\mathrm{CO}_{2}$ are described in Fig. 2 (CHO-TCA), Fig. 3 (PRO$\mathrm{NaOH}$ ), and Fig. 4 (CR). In strains 5-7 and 1-2-1, increases in carbohydrate and protein, and decreases in cell residue, accompanied increased macroconidia formation as it was induced by increasing $\mathrm{CO}_{2}$ tensions. Strains 1-1-2 and 1-3-1 were poorly stimulated to form macroconidia and did not show increases in carbohydrate. Under increased atmospheric tensions of $\mathrm{CO}_{2}$, strain 1-1-2 increased in protein and decreased in cell residue; strain 1-3-1 did not increase in protein or decrease in cell residue. Paper chromatograms of TCA extract hydrolysates from all cultures showed that glucose was the predominant sugar in these fractions; traces of galactose and the unidentified sugar were also present. When strain 5-7 was grown on Sabouraud's conservation medium under increased tensions of $\mathrm{CO}_{2}$, macroconidia formation was poorly stimulated. Analyses of gross chemical components during incubation with $\mathrm{CO}_{2}$ revealed no increase in carbohydrate, an increase in protein, and no decrease in cell residue.

\section{DISCUSSION}

The formation of macroconidia and microconidia by Trichophyton mentagrophytes implies that differences in structure and function, as well as size, exist between the two spore forms. Unfortunately, information about functional differences between macroconidia and microconidia is not available. One difference in function has been implied (Wilhelm, 1947). Since macroconidia are multinucleate and microconidia are uninucleate (Emmons, 1934), a heterokaryotic state may exist in the multinuclear compartment of a macroconidium and provide a survival advantage by allowing the macroconidium to carry a recessive gene.

The stimulation of macroconidia formation by incubation in increased $\mathrm{CO}_{2}$ tensions is difficult to investigate, since very little is known about the physiology of the organism. If any existing differences in the gross chemical components of morphological structures were known, it should be possible to investigate the physiological changes in mycelia during the process of sporulation and the effect of $\mathrm{CO}_{2}$ on these changes. The experiments described in this paper were designed to this end.

It was previously demonstrated that stimulation of macroconidia formation by incubation in increased $\mathrm{CO}_{2}$ tensions did not occur when glucose was omitted from the medium. This requirement for glucose may be a direct reflexion of the higher concentration of carbohydrate in macroconidia than in microconidia or hyphae. Perhaps the formation of macroconidia requires higher concentrations of glucose in the medium for a structural precursor of the spore itself. The carbohydrate appears to reside in the cytoplasm.

It is our experience that macroconidia germinate more rapidly than microconidia on Sabouraud's glucose agar. Wheeler, Cabaniss \& Cawley (1958) showed that macroconidia of Microsporum fulvum, another dermatophyte, were capable of germinating in distilled water in $4.5-10 \mathrm{hr}$. (confirmed in our laboratory). Macroconidia of 
Trichophyton mentagrophytes germinate in distilled water in $8 \mathrm{hr}$. when they are aerated; microconidia will not germinate under the same conditions. We have postulated that the higher concentration of carbohydrate in macroconidia may be more available as an energy source for germination than the lower quantity in microconidia. This is not the only factor to be considered, however, since microconidia suspended in $4 \%$ glucose solution will not germinate in $48 \mathrm{hr}$.

Other changes in gross chemical components of cultures also occur during incubation in increased $\mathrm{CO}_{2}$ tensions, particularly, in protein and cell residue fractions. However, investigations into these two fractions have been deferred because the separation of strong macroconidia-forming mutants and weak macroconidiaforming mutants is not as clear as in the carbohydrate fraction. These fractions have not, by any means, been dismissed from further consideration since the process of macroconidia formation must be complex. Physiological and structural changes during sporulation will rely heavily upon the formation of new and more protein. Generalizations about the sporulating processes of fungi are difficult to make. The factors which influence spore formation are many and varied (Cochrane, 1958); an agent which stimulates sporulation in one organism may inhibit it in another. Comparisons of sporulating processes will have to await more studies similar to those of Wright \& Anderson (1958) on Dictyostelium discoideum and Cantino (1956) on Blastocladiella emersonii. It is of interest that $\boldsymbol{D}$. discoideum accumulates cellulose during one phase of sporulation and that $\mathrm{CO}_{2}$ induces the formation of resistant sporangia in B. emersonii. Cantino \& Horenstein (1956) implicated a succinateketoglutarate-isocitrate cycle in the sporulation of Blastocladiella. Whether these aspects of carbohydrate metabolism are related to the accumulation of glucose in Trichophyton mentagrophytes remains to be seen.

This work was supported by Grant E-1201 from the National Institutes of Health, U.S.A.

\section{REFERENCES}

Cantino, E. C. (1956). The relation between cellular metabolism and morphogenesis in Blastocladiella. Mycologia, 48, 225.

Cantino, E. C. \& Horensteris, E. A. (1956). The stimulating effect of light upon growth and $\mathrm{CO}_{2}$ fixation in Blastocladiella. I. The S.K.I. cycle. Mycologia, 48, 777 .

ChIN, B. \& KNIGHT, S. G. (1957). Growth of Trichophyton mentagrophytes and Trichophyton rubrum in increased carbon dioxide tensions. J. gen. Microbiol. 16, 642.

Cochrane, V. W. (1958). Physiology of Fungi. New York: John Wiley and Sons, Inc.

Emmons, C. W. (1934). Dermatophytes. Natural groupings based on form of the spores and accessory organs. Arch. Derm. Syph. (Chicago), 30, 337.

Emmons, C. W. \& Hollaender, A. (1939). The influence of monochromatic ultraviolet radiation on the rate of variant production in Trichophyton mentagrophytes. Genetics, 24, 70.

KECH, K. (1958). An ultramicro technique for the determination of desoxypentose nucleic acid. Arch. Biochim. Biophys. 63, 446.

LoEwus, F. A. (1952). Improvement in anthrone method for colorimetric determination of carbohydrates. Anal. Chem. 24, 219.

Logan, J. E., Manneli, W. A. \& Rossiter, R. J. (1952). A note on the determination of deoxypentosenucleic acid and pentosenucleic acid in tissue from the nervous system by ultraviolet absorption. Biochem. J. 51, 480.

Lowry, O. H., Rosenbrough, W. J., Farr, A. L. \& Randall, R. J. (1951). Protein measurements with Folin phenol reagent. J. biol. Chem. 193, 265. 
Schneider, W. C. (1945). Phosphorous compounds in animal tissues. I. Extraction and estimation of deoxypentosenucleic acid and pentosenucleic acid. J. biol. Chem. 164, 293.

Sмптн, J. (1958). Chromatographic Techniques. Chemical and Biochemical Applications. New York: Interscience Publishers, Inc.

Umbreit, W. W., Burris, R. H. \& Stauffer, J. F. (1957). Manometric Techniques, 3rd ed. Minneapolis, Minn., U.S.A.: Burgess Publishing Co.

Wheeler, C. E., Cabaniss, W. H. \& CawLey, E. P. (1958). The germination of fuseaux of Microsporum fulvum. J. invest. Derm. 30, 27.

Wrreetm, S. (1947). The dual phenomenon in the dermatophytes. Mycologia, 39, 716.

Wright, Barbara E. \& Anderson, Minnie L. (1958). Enzyme patterns during differentiation in the slime mold. In The Chemical Basis of Development, p. 206. Ed. by W. D. McElroy and B. Glass. Baltimore, Maryland: Johns Hopkins Press. 